\title{
Wirelessly activated device with integrated ionic polymer metal composite (IPMC) cantilever valve for targeted drug delivery
}

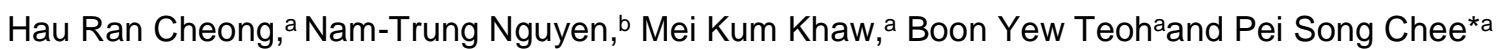 \\ Lee Kong Chian Faculty of Engineering and Science, Universiti Tunku Abdul Rahman, Bandar Sungai Long, Kajang 43000, \\ Selangor, Malaysia. \\ Queensland Micro-and Nanotechnology Centre, Griffith University, Nathan QLD 4111, Australia. \\ Email: cheeps@utar.edu.my \\ This paper reports a wirelessly powered ionic polymer-metal composite (IPMC) soft actuator operated by external radio frequency \\ (RF) magnetic fields for targeted drug delivery. A $183 \mu \mathrm{m}$ thick IPMC cantilever valve was attached with an embedded LC \\ resonant circuit to wirelessly control the actuator when the field frequency is tuned to its resonant frequency of approximately 25 \\ $\mathrm{MHz}$. Experimental characterization of the fabricated actuator showed a cumulative cantilever deflection of $160 \mu \mathrm{m}$ for three \\ repeated RF ON-OFF cycles at $0.6 \mathrm{~W}$ input power. The device was loaded with dye solution and immersed in DI water to \\ demonstrate wireless drug release. Qualitative result shows the successful release of the dye solution from the device reservoir. \\ The release rate can be controlled by tuning the RF input power. We achieved a maximum average release rate of $\sim 0.1 \mu \mathrm{l} / \mathrm{s}$. We \\ further conducted in-vitro study with human tumor cells (HeLa) to demonstrate the proof of concept of the developed device. The \\ experiments show promising results towards the intended drug delivery application.
}

\section{Introduction}

Recent advances in micromachining and 3D nanoprinting technology ${ }^{1,2}$ lead to extensive research and development activities of wirelessly controllable actuators. These actuators are particularly suitable for implantable drug delivery devices, which allow drugs to be provided precisely at a specific site. These devices allow automated drug release at an efficacious location to maximize the effectiveness of drug therapies such as epilepsy treatment, ${ }^{3}$ chronic diseases, ${ }^{4,5}$ osteoporosis treatment, ${ }^{6}$ cardiac resuscitation, ${ }^{7}$ cancer radiation reduction $^{8}$ as well as diabetic retinopathy ${ }^{9}$. Early approaches of wireless drug delivery use actuators ${ }^{10-12}$ that require additional components, control circuitry and batteries to trigger drug release. However, this approach makes the device bulky and more invasive. Moreover, limited battery life is detrimental to patient's health. Different types of wireless thermal microactuators for drug delivery applications have been reported, including shape memory polymer (SMP), ${ }^{13}$ hydrogel ${ }^{14}$ and thermopneumatic actuators. ${ }^{15}$ Although drug can be precisely delivered to the targeted cells, the thermal actuators might cause tissue damage and therefore, not well suited for implantable devices. In addition, changes in the in-vivo environment might deteriorate the actuator performance. Low-voltage actuation may be a feasible approach for implantable devices to overcome the heating problem.

Ionic polymer metal composite (IPMC) actuator is a promising candidate that can be operated under a relatively low voltage and without induced heat. IPMC actuator is an electroactive polymer (EAP) that eliminates the need for complex fabrication process. The actuator consists of a thin ion exchange polymer membrane sandwiched between two metal electrodes. This actuator provides a fast response and can be easily miniaturized.16 To broaden the actuator's mobility, wireless powered IPMC has been extensively investigated. Lee et al. reported a $9 \mathrm{GHz}$ microwave powered IPMC actuator using microstrip patch antenna patterned on the surface of the IPMC. ${ }^{17}$ This approach, despite eliminating battery involvement, is sensitive to the environmental changes, i.e. temperature and humidity. ${ }^{18}$ Additional circuitry such as band pass filter is required to eliminate the external interference. Low-frequency wireless radio frequency (RF) magnetic field power transfer offers the greatest potential to power the IPMC as it has lower power consumption and is relatively stable. One of such applications with wireless power supply was reported by K. Abdelnour etal. with magnetic coupled inductor-capacitor (LC) receiver coil for underwater robotic microswimmers. ${ }^{19}$ The power was transferred wirelessly to the LC coaxial coil, when its frequency was tuned to the same resonant frequency as the external power source. However, the device tends to have a larger footprint and has limited its practicability in packaging.

There have been some development efforts using IPMC soft actuator for controlled drug delivery. The biocompatible and soft muscle-like actuation makes this actuator well suited for wearable or implantable devices. ${ }^{20}$ Active-pumping drug delivery device ${ }^{21,22}$ offers high drug release rate and controlled pulsatile dosage. In these reports, the oscillatory movement of the IPMC based membrane creates a low pressure to draw the fluid into the pumping chamber and a high pressure to force the fluid out to the pump outlet. This drug release was constrained by the stroke formation of the IPMC membrane and prone to device failure. 
Moreover, the design poses the risk of incomplete drug release from the reservoir. Recently, Mousavi et al. reported a remotely controlled IPMC capping layer for triggering the opening of an orifice to induce the drug release through diffusion. ${ }^{23}$ Although the drug release can be triggered wirelessly, the diffusive release profile limits the precise dosage control. In addition, the use of silicon as device material required a complex fabrication and LC integration process. To our best knowledge, only this work reported on the application of IPMC for drug delivery in a wireless manner; there is no experimental study on the behavior of the cells with the released drug.

The present paper reports a potential implantable drug delivery device using a soft cantilever valve that is made of ionic polymer-metal composite (IPMC) actuator with an integrated planar LC receiver circuit. Under an applied voltage, the developed cantilever valve deflects to activate the drug release mechanism. This voltage is generated, when the external radio-frequency (RF) magnetic field matches the resonant frequency of the LC receiver circuit. Resonant coupling is chosen as the wireless powering scheme due to its robust performance. Advantages such as the long wireless transmission distance have been discussed in our previous work. ${ }^{24}$ Systematic in vitro study demonstrates the reduction of HeLa cell viability through the release of Cisplatin drug from the fabricated prototype. These results demonstrated the proof of concept of our wirelessly activated soft IPMC cantilever valve for mobile, reliable and safe targeted drug delivery.

\section{Device design and working principle}

The design of the developed drug delivery device is depicted in Fig 1(a, b). The device body was made of biocompatible polydimethylsiloxane (PDMS). ${ }^{25}$ It is designed to have overall dimension of $15 \times 25 \times 6 \mathrm{~mm}^{3}$, including a drug reservoir with maximum loading capacity $520 \mu \mathrm{L}$. The wireless power transfer setup utilized a planar inductor-capacitor (LC) resonant circuit that serves as the wireless receiver to activate the IPMC cantilever and an external transmitter circuit. The LC receiver circuit consists of two rectangular-spiral coils as the resonator and load loops or source loops with integrated capacitor plates to form an LC tank, fabricated using printed circuit board technology. The inductances of the square loop pattern in both circuits are expressed by Modified Wheeler Formula:

$$
L=K_{1} \mu_{0} \frac{n^{2} d_{a v g}}{1+K_{2 \rho}}
$$

where $\rho$ is the fill ratio, $n$ is the number of turns, $K_{1}$ and $K_{2}$ are layout coefficients, respectively. The outer coil diameter is defined as $d_{\text {out }}$ and the inner diameter is defined as $d_{i n}$. To calculate the average diameter,

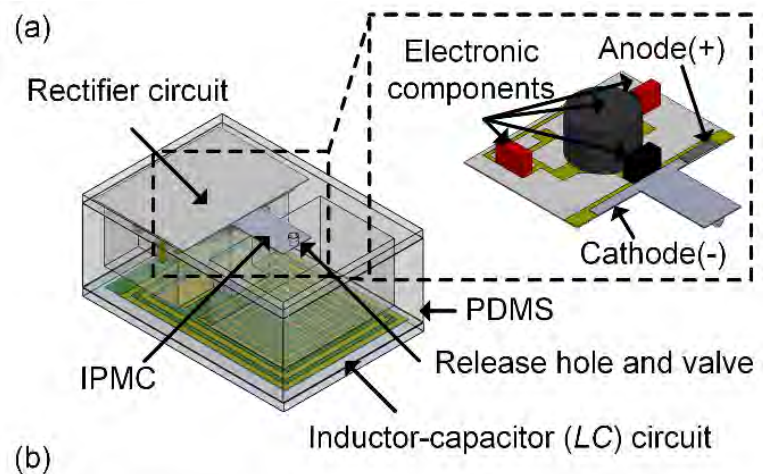

(b)

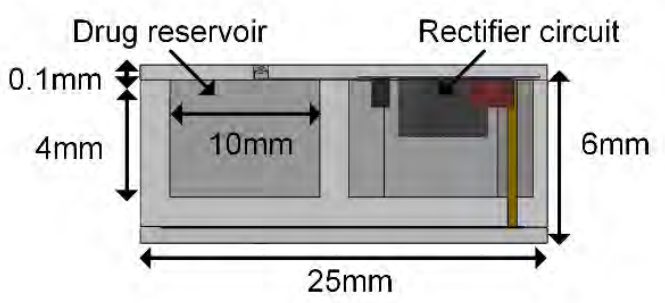

Fig.1 IPMC-based cantilever valve for drug delivery. (a) Schematic illustration of the drug delivery device. (b) Side-view of the device. 
$d_{\text {avg }}=0.5\left(d_{\text {out }}+d_{\text {in }}\right)$. The resonant frequency of the LC tank of the both circuits can be determined using the following equation:

$$
f=\frac{1}{2 \pi \sqrt{L C}}
$$

The LC tank is coupled with a DC rectifier circuit, fabricated from a single-sided copper-clad polyimide (PI) film with $0.11 \mu \mathrm{m}$ thickness. The LC receiver circuit is encapsulated at the bottom of the device body with 0.1 mm thick PDMS layer. A square cavity is created at the pump body to accommodate a DC rectifier circuit with integrated IPMC-based cantilever to function as a wireless valve to control the opening gap over the release orifice of the drug delivery device. The PDMS layer isolates the contact point between the cantilever and DC rectifier circuit, ${ }^{26}$ preventing galvanic corrosion that may attack the electrodes while operating in an aqueous surrounding.

Operation of the device is controlled by wirelessly resonating the LC tank. The load loops induce an AC voltage through faradays law, ${ }^{23}$ when it is exposed to the external magnetic field generated by the external transmitter circuit at its resonant frequency. The cantilever bends when the induced DC voltage from the DC rectifier circuit rises above a critical value, due to the migration of mobile cations in the IPMC material to the cathode, as shown in Fig 2(a). This phenomenon creates a pressure across the cantilever and bends it toward the anode. ${ }^{27}$ This deflection opens the release orifice and creates pressure inside the reservoir to pump the drug out. The rate of drug release can be wirelessly controlled by manipulating the bending force through tuning the RF input power from the external transmitter. Increasing the RF power increases the bending displacement of the IPMC-based cantilever, leading to a larger pressure induced.

An analysis using COMSOL Multiphysics optimized the design dimension to provide sufficient displacement to move the valve away from the release orifice. The numerical model can predict the displacement and the temporal response of the IPMC-based cantilever valve. In the simulated model, the time-dependent transport of the cation in the IPMC-based cantilever is described using Nernst-Plank equation.

$\frac{\partial \mathrm{C}}{\partial \mathrm{t}} \partial \mathrm{t}+\nabla \cdot(-\mathrm{D} \nabla \mathrm{C}-\mathrm{z} \mu \mathrm{FC} \nabla \phi-\mu \mathrm{C} \Delta \mathrm{V} \nabla \mathrm{P})=0$

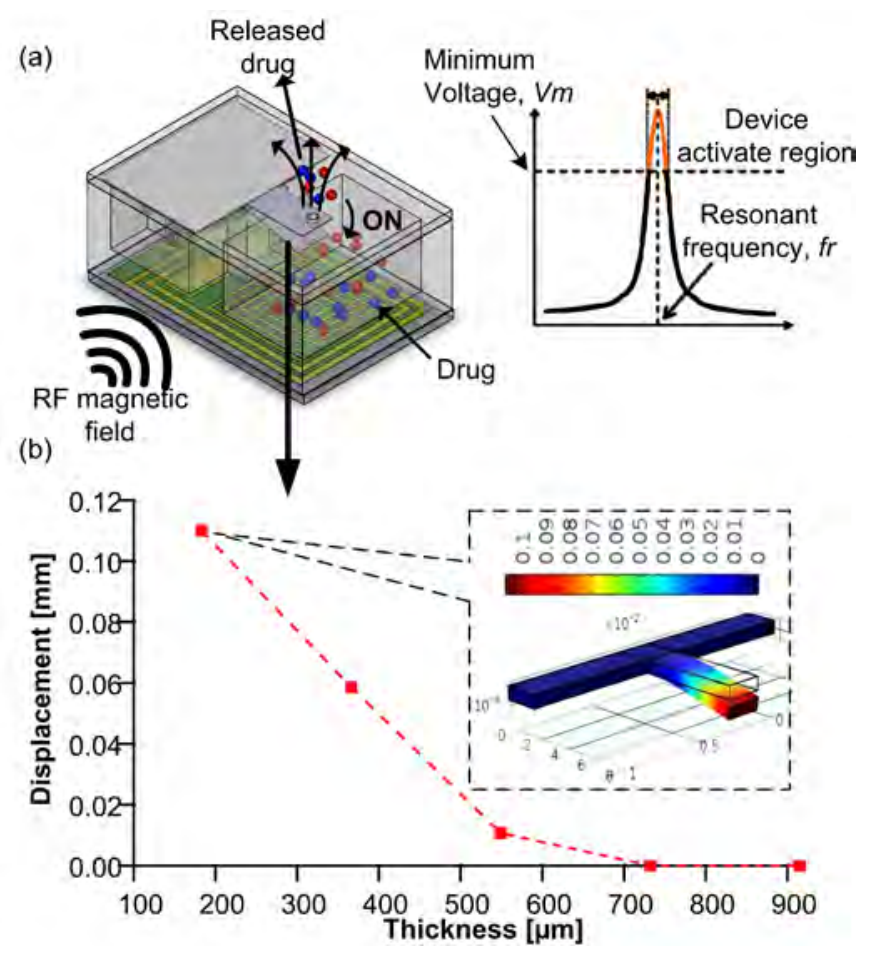

Fig.2 (a) Conceptual diagram and frequency-sensitive working principle of the RF powered device. (b) Displacement versus thickness of the IPMC layer. The inset illustrates the simulation result using COMSOL Multiphysics. 


$$
\begin{aligned}
& j_{\text {diffusion }}=-\mathrm{D} \nabla \mathrm{C} \\
& j_{\text {migration in electric field }}=-\mathrm{z} \mu \mathrm{FC} \nabla \varphi(5) \\
& j_{\text {convection }}=\mathrm{uC}
\end{aligned}
$$

where $\mathrm{D}$ is the diffusion constant, $\mathrm{C}$ is the cation concentration, $\mu$ is the mobility of cations, $\mathrm{F}$ is the Faraday constant, $\mathrm{z}$ is the charge number, and $\varphi$ is the electric potential in the polymer. The cation velocity, $u$, can be expressed in terms of the solvent pressure gradient as:

$\mathrm{u}=-\mu \Delta \mathrm{V} \nabla \mathrm{P}$

where $\Delta \mathrm{V}$ is the molar volume that quantifies the cation hydrophilicity and $\mathrm{P}$ is the solvent pressure. Fig. 2(b) shows the deflection of the cantilever as a function of the IPMC layer thickness under an applied DC voltage of $2 \mathrm{~V}$. A single Nafion layer or a thickness of $183 \mu \mathrm{m}$ results in the largest displacement. Increasing thickness leads to decreasing displacement due to the increasing stiffness of the cantilever. The inset in Fig. 2(b) shows a maximum displacement of $0.11 \mathrm{~mm}$, in good agreement with experimental data.

\section{Device fabrication and experiment setup}

The construction of the drug delivery device followed three main steps, i.e., LC circuit fabrication, integration of the IPMC cantilever to the fabricated DC rectifier circuit and the fabrication of the pump body. Fig 3(a) shows the fabrication and integration processes for the fabrication of the drug delivery device. First, a double sided Cu-clad PI film (AP7156E, DuPont Pyralux Polyimide Film, USA) was patterned with two rectangular-spiral coils as the resonator and load loop and a capacitor plate via dry film photolithography process. Next, both top and bottom sides of the $L C$ receiver circuit undergo etching process to remove the copper-clad layers. Vial contacts were etched at the PI film and sealed with silver conducting epoxy to connect the top and bottom copper side of the PI film. Subsequently, a $0.11-\mathrm{mm}$ thick DC rectifier circuit was fabricated using the similar method, with bonded power electronic components to form class-D half wave rectifier circuit. The fabricated IPMC was cut into T-shape to form a 8-mm long cantilever and bonded to the DC rectifier circuit. The electrical contact between the LC receiver circuit and DC rectifier circuit was achieved using silver conducting epoxy. Both circuits were coated with polydimethylsiloxane (PDMS) layer (Sylgard 184, Dow Corning Corp, USA) (mixed with the ratio of 1: 10) to make the surface electrically isolated and biocompatible.

The IPMC-based cantilever valve was fabricated from Nafion-117 film (Dupont, USA) of $183 \mu \mathrm{m}$ thickness. The cantilever valve was cut into the desired dimension and shape prior to the blasting process to increase the surface area density ${ }^{28}$ of the electrodes. After thorough cleaning in a mixture of sulphuric acid $\left(\mathrm{H}_{2} \mathrm{SO}_{4}\right)$ and hydrogen peroxide $\left(\mathrm{H}_{2} \mathrm{O}_{2}\right)$, the anode and cathode electrode pads were photo lithographically patterned on the Nafion-117. The silver layer was then plated on the both Nafion-117 surfaces (top and bottom) using silver mirror electroless plating process by dipping the Nafion-117 into a complex solution of $\left[\mathrm{Ag}\left(\mathrm{NH}_{3}\right)_{2}\right] \mathrm{OH}$, mixture of $1.6 \mathrm{~g}$ sodium hydroxide, $1.2 \mathrm{~g}$ silver nitrate, $2 \mathrm{~g}$ ammonia solution (28\%) and $120 \mathrm{~g}$ DI water for 5 minutes. The Nafion-117 film was later transferred to another solution (mixture of $0.8 \mathrm{~g}$ glucose and $120 \mathrm{~g}$ DI water) for 5 minutes. The silver electrodes were formed in the following chemical reactions:

$$
\begin{aligned}
& 2 \mathrm{AgNO}+2 \mathrm{NaOH} \rightarrow \mathrm{Ag}_{2} \mathrm{O}+2 \mathrm{NaNO}_{3}+\mathrm{H}_{2} \mathrm{O} \\
& \mathrm{Ag}_{2} \mathrm{O}+4 \mathrm{NH}_{3}+\mathrm{H}_{2} \mathrm{O} \rightarrow 2\left[\mathrm{Ag}\left(\mathrm{NH}_{3}\right)_{2} \mathrm{OH}+2 \mathrm{H}_{2} \mathrm{O}\right. \\
& \mathrm{CH}_{2} \mathrm{OH}(\mathrm{CHOH})_{4} \mathrm{CHO}+2\left[\mathrm{Ag}\left(\mathrm{NH}_{3}\right)_{2}\right] \mathrm{OH} \rightarrow 2 \mathrm{Ag}+\mathrm{CH}_{2} \mathrm{OH}(\mathrm{CHOH})_{4} \mathrm{COONH}_{4}+3 \mathrm{NH}_{3}+3 \mathrm{H}_{2} \mathrm{O}
\end{aligned}
$$

Later, the pump body was constructed by pouring the same PDMS solution to a 3-D printed mold (using B9 creator, USA) and cured at room temperature for 48 hours. Finally, the LC receiver circuit and DC rectifier circuit were bonded to the constructed pump body with a punched 1-mm release orifice. The LC receiver circuit is integrated as a wireless receiver into the IPMC-based cantilever using chemical deposition on the photo lithographically patterned Nafion 117. The process forms different polarity electrode pads and is bonded to the DC rectifier. This technique allows for a small device footprint, in contrast to the previously reported bulky devices with external clamps as electrode. ${ }^{29}$ Fig. 3(b) shows the fabricated drug delivery device and the scanning electron microscopy (SEM) image of the fabricated IPMC-based cantilever with the silver electrode layer. 
(a)

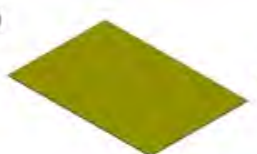

(i)

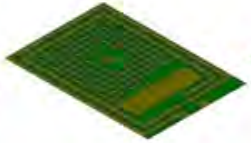

(ii)

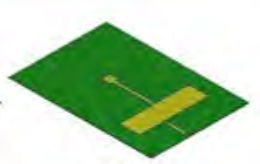

(iii)

LC receiver circuit

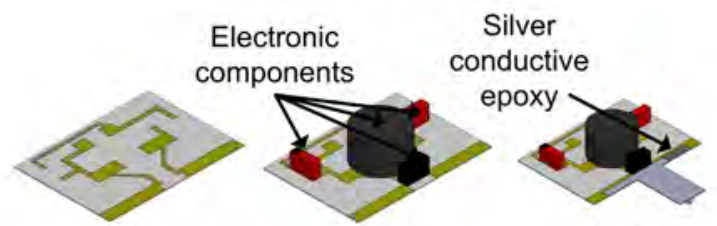

(iv)

(v)

(vi)

Rectifier circuit integrated with IPMC

(b)
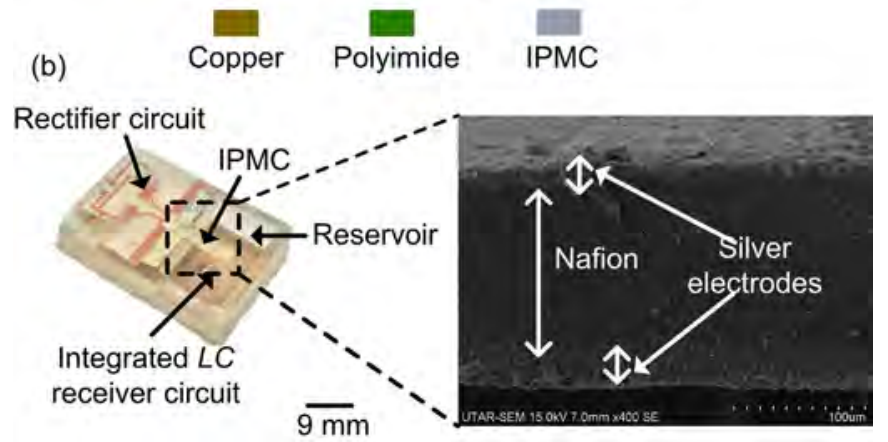

Fig.3 (a) Device fabrication process, (i) double layer Cu-clad sheet, (ii) forming of inductor and capacitor plate (top view), (iii) forming of capacitor plate (bottom-view), (iv) etching of the rectifier circuit, (v) soldering of the electronic components and (vi) bonding the IPMC cantilever with rectifier circuit. (b) Complete device and scanning electron microscopy (SEM) image of a cross-section of the IPMC layer.

Demonstration of wirelessly controlled drug delivery was performed with the setup consisting of a square wave function generator (SFG-830, GW Instek, Taiwan) and a class-E power amplifier (output power of $0.65 \mathrm{~W}$ ) that produced the magnetic field through the external transmitter connected to the amplifier. An oscilloscope (MDS1102CA, Matrix, China) was used to determine the electrical response of the LC receiver circuit. The setup also involved a laser displacement sensor (CD5-85, Optex, Japan) and a load cell (FX300i, A\&D, Japan) to characterize the displacement and force of the cantilever valve, respectively. As a preliminary experiment, the fabricated LC receiver circuit was examined for its frequency dependency, separation distance and tilting alignment with external transmitter. Wireless release test for visual demonstration were conducted by loading the device's reservoir with food dye solution. The device was immersed in a beaker filled with DI water and then placed on top of the external transmitter. The device was then activated by radiating an RF field from the external transmitter (ESI, Fig. S1). Similar setup was utilized to measure the quantitative release of drug concentration from the developed device to an aqueous environment. This time, the device reservoir was filled with fluorescent solution (Sigma Aldrich, USA) and the liquid released was evaluated by a well-plate reader. In-vitro experiment was carried out by treating cervical tumor cells (HeLa Cells) with cisplatin to demonstrate the proof of concept of the developed device for drug delivery application. For the cell cultured experiment, both sterilized devices were loaded with Cisplatin and submerged in petri dishes filled with human cervix carcinoma cell line (HeLa) culture medium (mixture of 90\% RPMI (Sigma Aldrich, USA) and $10 \%$ of fetal bovine serum (FBS) (Sigma Aldrich, USA). RF field was activated at one petri dish whereas another petri dish serves as a control sample (RF turned off). Both cell culture media were collected after 10 minutes of RF activation and transferred to 96 well-plates (Greiner 96 Flat Bottom Transparent Polystyrene, Sigma Aldrich, USA), which have HeLa cells cultured at a seeding density of 7000 cells/well. To examine the cell viability, $10 \%$ concentration of MTT assay (Sigma Aldrich, USA) was added into the well with the volume $10 \mu \mathrm{l}$ and incubated in a 5\% CO2 incubator (HERAcell 150, Thermo (Heraeus), Germany) at $30^{\circ} \mathrm{C}$ for 1,3 and 24 hours. All experiments were carried out in the Biosafety cabinet (BIO II A, Telstar, Spain). 


\section{Results and discussion}

\subsection{Characterization of the fabricated LC receiver circuit}

The drug delivery device is activated once it is exposed to the external electromagnetic field of the transmitter circuit when the field frequency matches the resonant frequency of the LC receiver circuit. As discussed in the previous section, the LC receiver circuit is encapsulated with a PDMS layer $(\sim 0.02 \mathrm{~mm}$ thick) in the device package to comply with biocompatibility criteria. The impact of the PDMS layer on the resonant frequency of the LC receiver circuit was first examined. To determine the resonant frequency, the $S_{11}$ parameters of the two LC receiver circuits, with and without the PDMS layer, were measured using a vector network analyzer (VNA) (Rohde \& Schwarz, Germany) via inductive coupling (ESI Fig. S2) in air and in DI water. ESI Fig. S3 shows that the thin PDMS layer does not create any significant change to the resonant frequency of LC receiver circuit. Both samples exhibit identical resonant frequency of approximately $25 \mathrm{MHz}$ when measured in air and coupled with the DC rectifier circuit. To evaluate the effect of working environment on the resonance (ESI, Fig S4), the LC receiver circuit covered with PDMS was tested in DI water, air and skin mimicking gels (with and without muscle and fat gel models). The resonant frequency in these media exhibits an almost identical response, while different values of phase dip (Fig 4(a)) were observed in water and air. The PDMS encapsulation of the LC receiver circuit isolates the LC coil from the operating media ${ }^{30}$ and hence maintaining its inductance, in contrast to the device reported by ${ }^{31}$ with the resonant frequency shifted when changing from water and air. The difference value of the phase dip in water and air might be caused by the higher power absorption of water. ${ }^{32}$ This test proved that the fabricated LC receiver circuit suits well for implantation and may work in different media. 
The output voltage of the LC receiver circuit also depends on the coupling mechanism of the transmitter circuit. The size of transmitter coil is designed to have large dimension to enhance the power transfer efficiency. Experiments were conducted to study the behavior of the output voltage of the LC receiver circuit (after feeding to the DC rectifier circuit) at different placement position on the transmitter circuit, which has a receiver-transmitter separation distance of $1.5 \mathrm{~cm}$. Fig. $4(\mathrm{~b})$ shows that the LC receiver circuit achieves a large voltage response of approximately $2.9 \mathrm{~V}$, when it is placed at the center of the transmitter circuit. The voltage decreases with increasing distance between the LC receiver circuit and the center of the transmitter circuit. Understanding the interaction between the transmitter and the LC receiver circuit is important for an optimum power transfer. Thus, the maximum power transfer distance and the orientation of the LC receiver circuit were optimized. The result presented in Fig. 4(c) shows a gradual decrease of the DC voltage with increasing distance between the $\mathrm{LC}$ receiver circuit and the transmitter, due to less receiving power. This result suggests that the maximum separation distance between the transmitter circuit and LC receiver circuit is about $4.5 \mathrm{~cm}$ to produce an output voltage of approximately $1 \mathrm{Vdc}$, which is the minimum voltage to activate the IPMC-based cantilever. Fig. 4(d) shows the response of the $\mathrm{LC}$ receiver circuit at different alignment angles. The maximum voltage of approximately $3.1 \mathrm{Vdc}$ was achieved with a parallel alignment ( 0 degree from the transmitter). This trend is consistent with the fact that the maximum separation distance highly depends on the magnitude of the cutting flux. There is no cutting magnetic flux when the LC receiver circuit is placed perpendicular to the transmitter circuit.

(a)

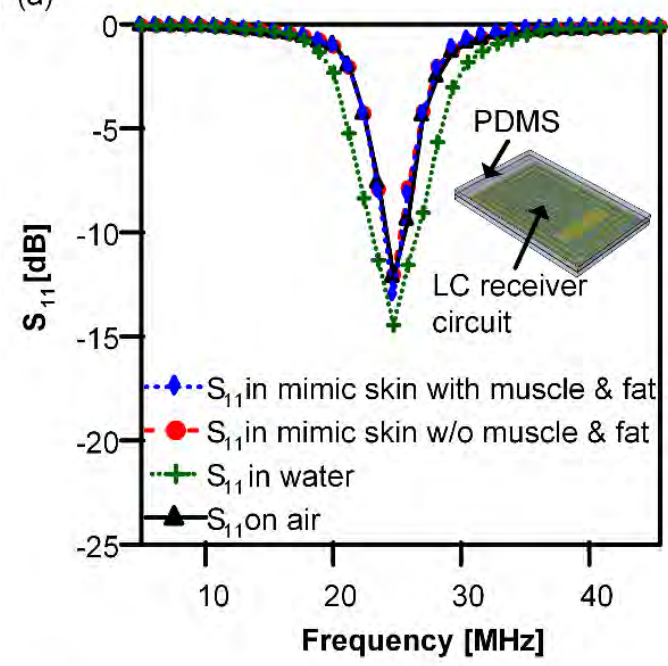

(c)

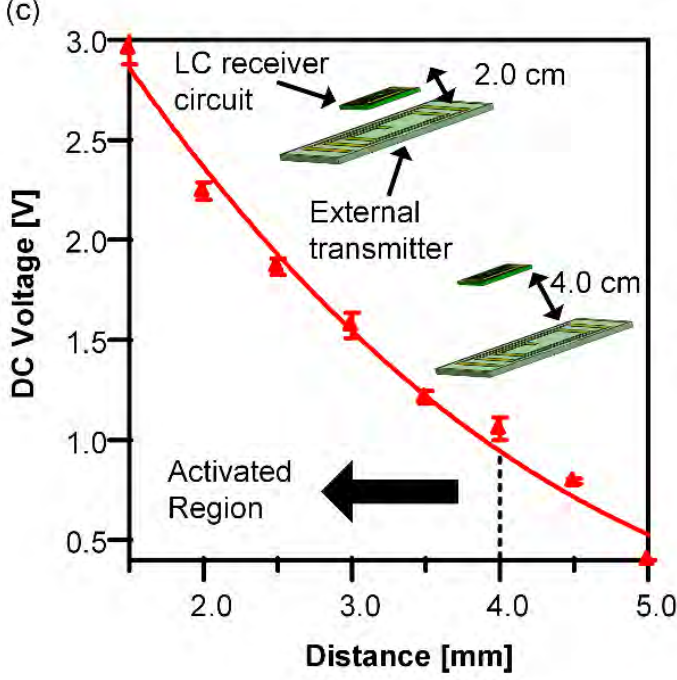

(b)

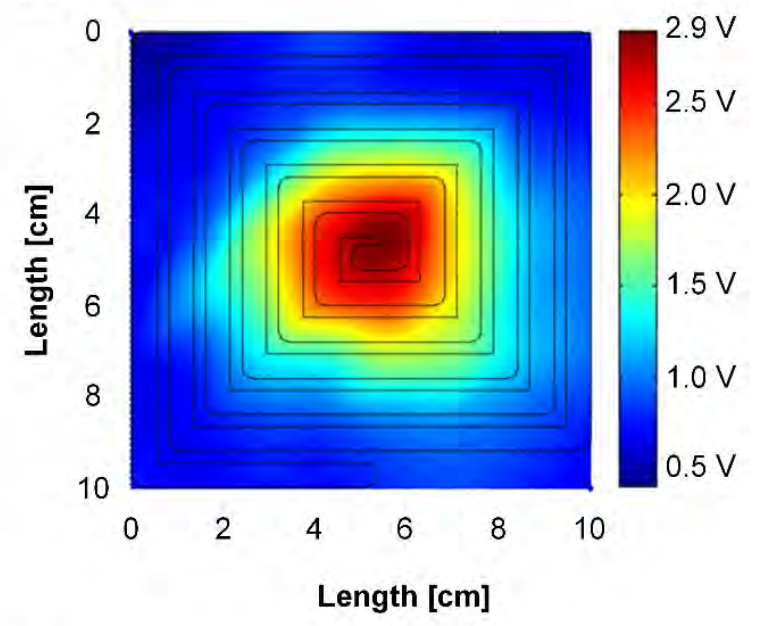

(d)

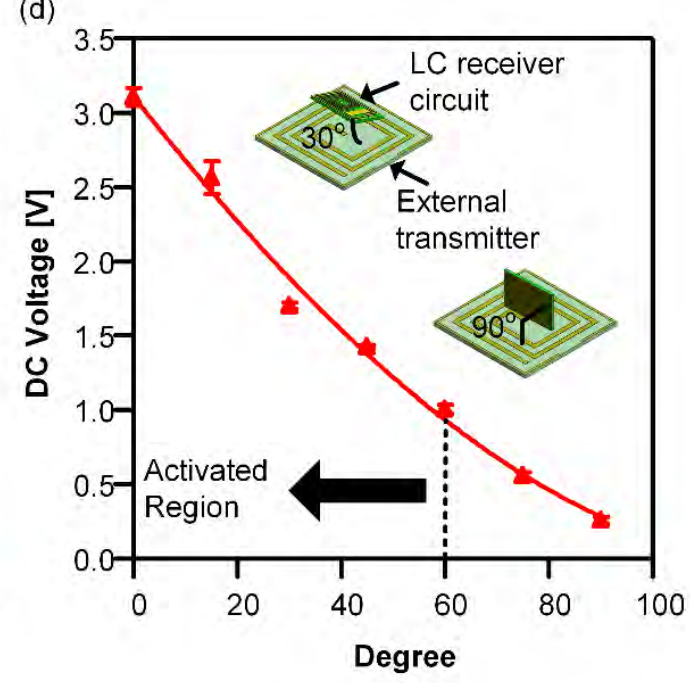

Fig. 4 Interaction behavior of a LC receiver circuit and the external transmitter. (a) S11 parameter of the LC receiver circuit in air, water and skin mimicking gels (with and without muscle and fat gel models). (b) Coupling response of the LC receiver circuit due to different placement on the transmitter at $1.5 \mathrm{~cm}$ separation distance (after fed to a DC rectifier circuit). (c) DC voltage versus distance between LC receiver circuit and the external transmitter circuit. (d) DC voltage versus tilting angle of $L C$ receiver circuit. 


\subsection{Wireless test of the IPMC-based cantilever valve}

IPMC-based cantilever consists of an ion exchange sheet clamped with two silver electrode plates, behaving as a capacitor in a lumped mass model. ${ }^{19}$ An impedance analysis was conducted to identify the capacitor behavior of the IPMC-based cantilever with a $10 \Omega$ shunt resistor connected in series at multiple wireless DC step voltage of $1 \mathrm{~V}$, $2 \mathrm{~V}$ and $3 \mathrm{~V}$ (maximum DC voltage from the DC rectifier circuit). The current induced at the IPMC-based cantilever

(a)

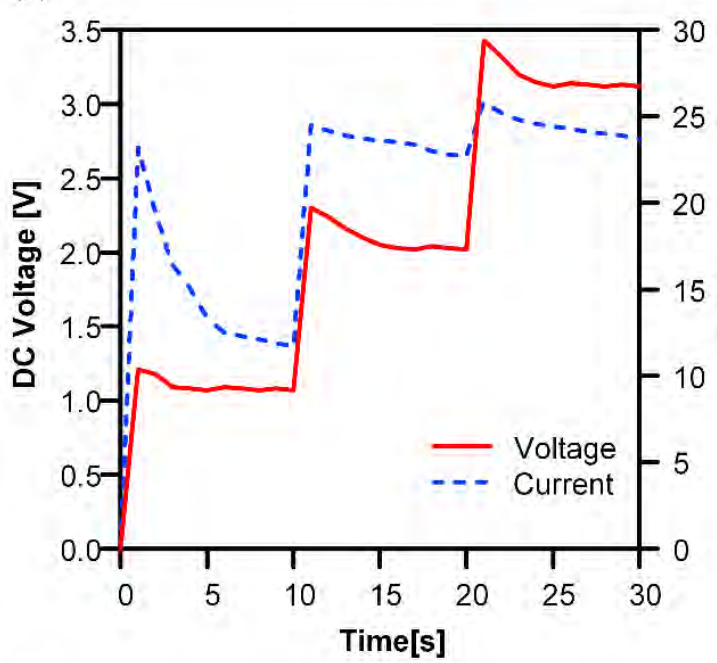

(c)

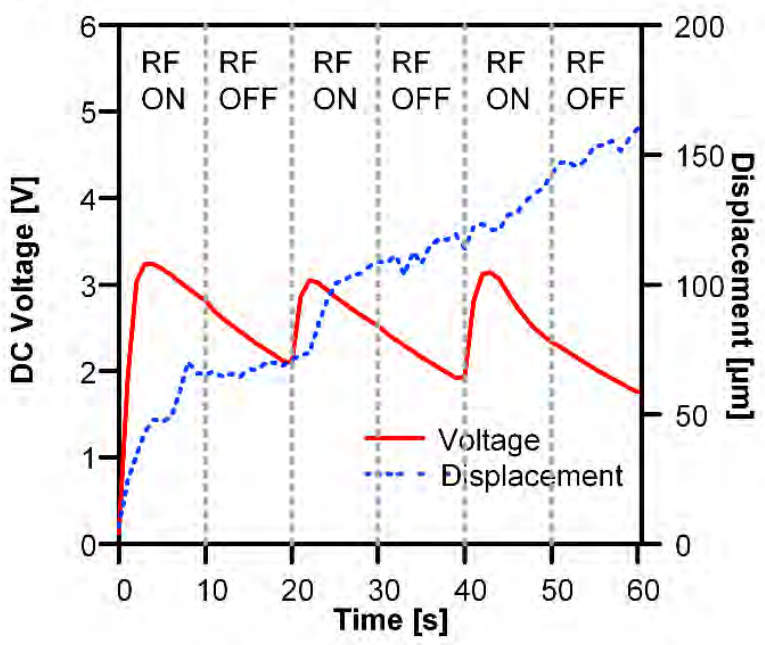

(b)

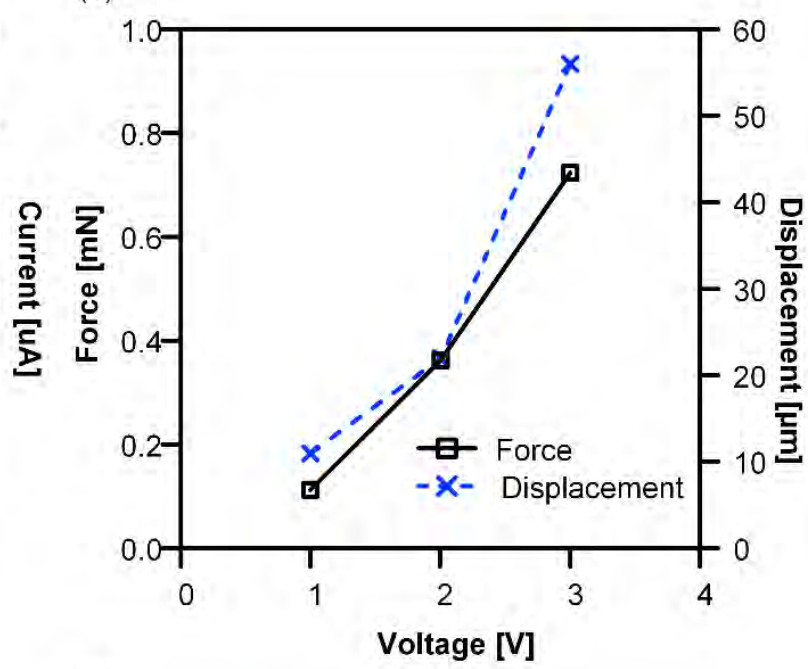

(d)

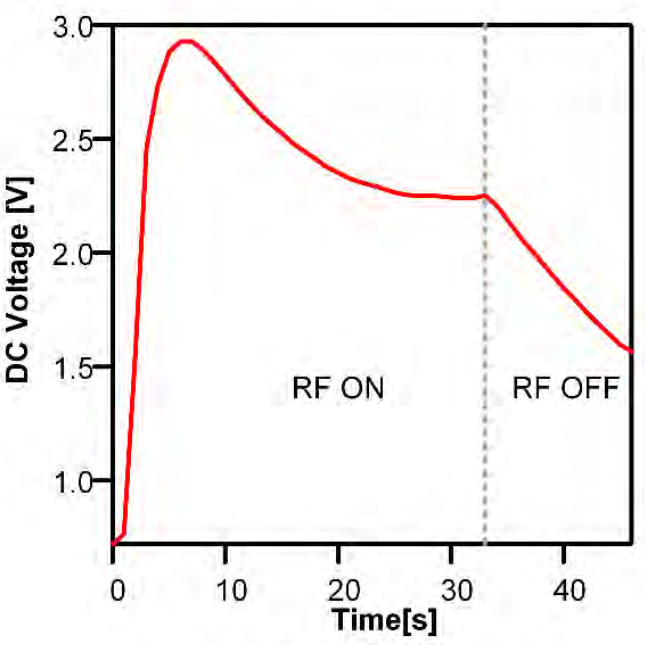

Fig.5 Wireless characterization of the IPMC cantilever valve. (a) Current response of the IPMC actuator with step voltage of $1 \mathrm{Vdc}, 2 \mathrm{Vdc} 3 \mathrm{Vdc}$. (b) IPMC actuator block force and tip displacement response with step voltage of $1 \mathrm{~V}, 2 \mathrm{~V}, 3 \mathrm{~V}$. (c) IPMC actuator tip displacement and DC voltage versus time with responded to $10 \mathrm{~s} R F \mathrm{ON} / 10 \mathrm{~s} R F$ OFF activation. (d) Voltage response of rectifier circuit activated in one RF ON/OFF cycle. 
(a)

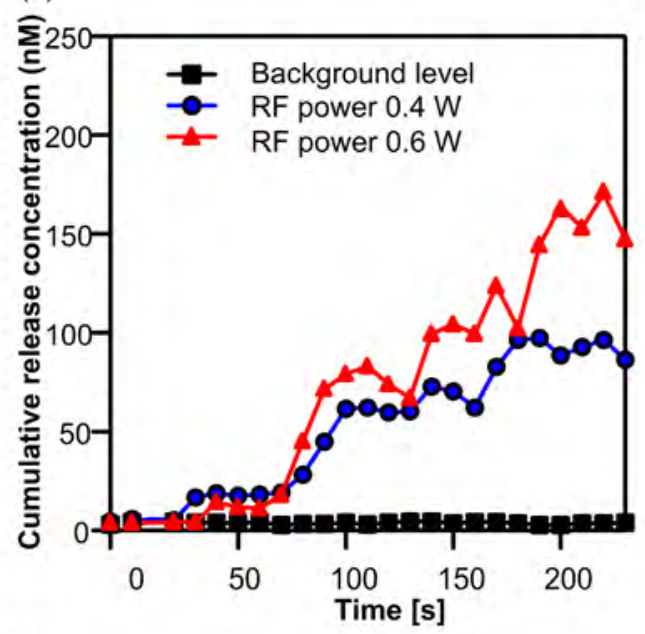

(c)

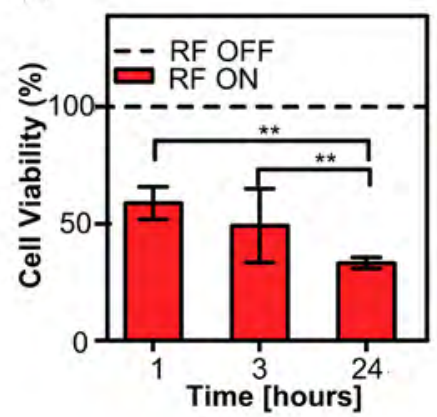

(b)

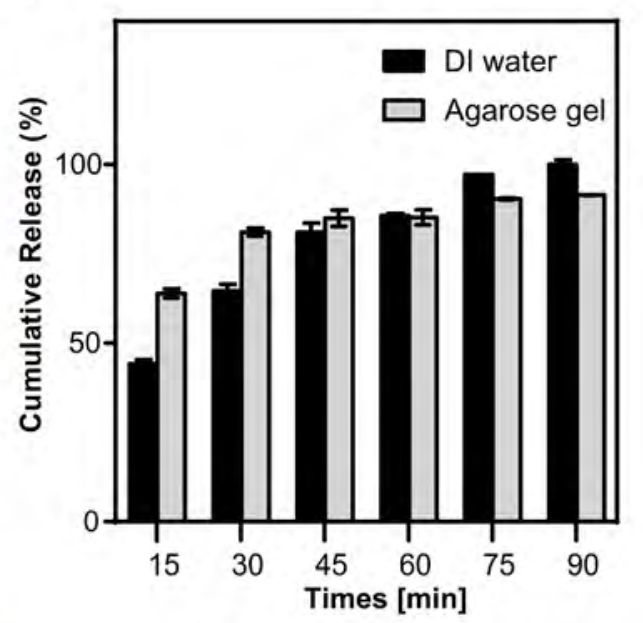

(e)

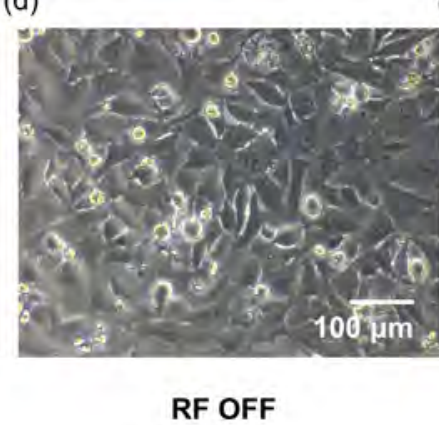

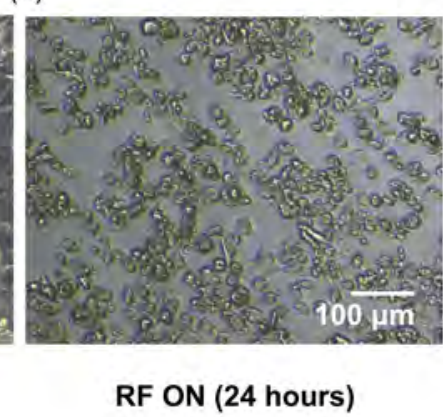

Fig.6 In vitro evaluations of the developed prototype in cell viability assay (MTT) using human tumor cells (HeLa). (a) Cumulative release of fluorescent concentration from the fabricated device at $0.4 \mathrm{~W}$ and $0.6 \mathrm{~W}$. (b) Percentage of fluorescent released from the device immersed in $0.2 \%$ agarose gel and DI water. (c) Cell viability assay using Cisplatin drug released at RF turned off and on at different incubation times (Data is average + SD of 3 independent experiments. Asterisks denote statistical significant difference as compared to cell viability at 24 hours (Student's T test, ${ }^{* *}$ p $<0.01$ ). (c) Optical microscope images (scale bar: $100 \mathrm{um}$ ) of Hela cells at (d) RF turned off and (e) RF turned on (24 hours incubation time) configuration.

was experimentally measured using a data acquisition module (Elvis board II, National Instruments, U.S). The current increases rapidly at each step voltage changes and slowly decreases to its steady state, Fig. 5(a). This can be explained from the lumped mass model, where the current (corresponding to the motion of cations in the IPMCbased cantilever) stop flowing when it reaches equilibrium. The charge stored in the IPMC cantilever is proportional to the produced deflection, and thus controlling the actuating behavior of the IPMC cantilever.

Fig. 5(b) shows that the IPMC cantilever tip displacement and block force increase with increasing applied voltage, measured by immersing the developed device in a beaker filled with DI water which was placed on top of the transmitter circuit. The measurement shows a deflection of approximately 11 um with the tip force of $0.1 \mathrm{mN}$ at $1 \mathrm{Vdc}$ induced voltage of the LC receiver circuit. Below $1 \mathrm{Vdc}$, the IPMC cantilever does not show any noticeable displacement as the low voltage is not able to initiate any cations' motion. The tip force increases to $0.36 \mathrm{mN}$ and $0.72 \mathrm{mN}$ for $2 \mathrm{Vdc}$ and $3 \mathrm{Vdc}$, respectively. It is worth noting that displacement of the IPMC cantilever can be improved by depositing a stressor layer on the cantilever surface. ${ }^{33,34}$

Fig. 5(c) shows the response of the IPMC actuator activated in 3 sets of repeated RF ON-OFF cycles: 10 seconds RF ON, 10 seconds RF OFF with 3 Vdc output voltages. A cantilever displacement of $66 \mu \mathrm{m}$ was measured at the 10th second of RF ON activation. The cantilever deflection, however, experiences a slight increase to $70 \mu \mathrm{m}$ at the end of the RF OFF cycle. This behavior repeats until the end of the experiment with the cumulative deflection values of $110 \mu \mathrm{m}$ and $160 \mu \mathrm{m}$ for the second and third cycle, respectively. To examine the increase of IPMC displacement phenomenon at each RF OFF cycle, the voltage profile at the IPMC actuator is shown in Fig. 5(c). The voltage slightly decreases after the 3th second at the first RF ON cycle due to the capacitive effect, Fig. 5(a). 
On the other hand, the voltage decreases abruptly at the RF OFF cycle to approximately $2 \mathrm{Vdc}$. Fig. 5 (d) shows the voltage time response of the rectifier circuit in one RF ON/OFF cycle, which verifies the capacitive properties in the DC rectifier circuit. Fig. 5(b) shows that the IPMC actuator deflects at approximately 2 Vdc and therefore resulting in increasing cumulative IPMC actuator displacement in the RF OFF cycle. IPMC actuator remains at its final position if no voltage is applied across the membrane. As a preliminary test of wireless drug release for visual verification, a prototype with a reservoir filled with the dye solution was placed in a water-filled beaker and then activated with a RF field from an external transmitter circuit. The dye solution released from the reservoir to the surrounding water was clearly observed, ESI Fig. S5. These experiments qualitatively validated the device's functionality in a surrounding liquid at $0.4 \mathrm{~W}$ RF power.

Table S1 in the ESI compares the total power consumption for wireless drug delivery actuation with that of other devices reported in the literature. In comparison with thermally activated drug delivery device, i.e. shape memory polymer $^{31}(5 \mathrm{~W})$ and shape memory alloy ${ }^{35}(1.1 \mathrm{~W})$ our solution offers a significantly lower power consumption of $0.4 \mathrm{~W}$ and higher flow rate of $3.6 \mu \mathrm{l} / \mathrm{min}$. Electrolytic driven actuator ${ }^{36}$ may operate with lower power consumption of $4 \mathrm{~mW}$. However, the flow rate output is relatively low $(0.011 \mu \mathrm{L} / \mathrm{min})$. The consistency of drug release highly depends on bubble generation rate, which has the risk of premature bubble collapse and recombination.

\subsection{In-vitro study}

The quantitative release of drug concentration from the developed device to an aqueous environment was tested by measuring the fluorescent solution (Sigma Aldrich, USA) for every 10 seconds using a well-plate reader. All the measurements were performed without agitation or forced flow. Prior to device activation, leak test was performed with the device fully filled with fluorescein and immersed in DI water without turning on the RF power. Fig. 6(a) shows no distinct increase in intensity for the mentioned period, suggesting there is negligible off-state leakage of fluorescein to the environment. The intensity increases in a cumulative and incremental manner at RF ON with the power of $0.4 \mathrm{~W}$ and $0.6 \mathrm{~W}$ at an average released rate of $\sim 0.06 \mu \mathrm{l} / \mathrm{s}$ and $\sim 0.1 \mu \mathrm{l} / \mathrm{s}$, respectively. The results clearly demonstrate that tuned RF activation of the device is effective in performing fluorescein released using the wireless approach.

Practical applications require robustness and capability of the developed device to operate effectively in tissuelike environment. We conducted an experiment by embedding the device in an agarose gel (Sigma Aldrich, USA). Agarose gel, known as flexible substrate with physical properties similar to extracellular matrix (ECM), ${ }^{37,38}$ has been widely used as tissue-mimicking material..$^{39-41}$ The intensity of the fluorescein, corresponding to the cumulative drug release (expressed in term of total percentage) from the device's orifice, was measured using a fluorescent microscope (Olympus BX41, Tokyo, Japan). Fig. 6(b) presents the temporal response of fluorescein released from our device immersed in $0.2 \%$ agarose gel and DI water. The agarose content was chosen based on its comparable properties to collagen type $\mathrm{I}^{37}$. The device was then activated wirelessly with a RF power of $0.6 \mathrm{~W}$. The concentration of fluorescein released in DI water increases over time. In the first 45 minutes, the fluorescein released in DI water was measured to be lower than that in agarose. We hypothesise that the released fluorescein presented at the measurement location diffuses more quickly to the surrounding water than to the highly viscose agarose gel. The fluorescein in the reservoir is completely released in surrounding DI water after 90 minutes. The percentage released in agarose gel was approximately $91 \%$, indicating the time required for full fluorescein released in agarose gel was longer than in DI water. The test clearly showed well-defined and predictable responses of our device in different surrounding media.

To demonstrate the proof of concept of wirelessly activated drug delivery, in-vitro experiment was carried out by treating cervical tumor cells (HeLa Cells) with cisplatin. Cisplatin is a chemotherapeutic drug for the treatment of cervical cancer. ${ }^{42-44}$ Fig. 6(c) illustrates the cell viability assay of the tumor cells with drug released from the developed prototype immersed in Fetal Bovine Serum with RF turned on for 10 minutes. The released drug solutions were collected 10 minutes after releasing the drug and transferred to 96-well plate. The results indicate that the viability of the HeLa cells decreases significantly 1 to 3 hours after the wirelessly triggered release, followed by gradually reduction to $\sim 30 \%$ cell viability at 24 hours. Our developed prototype does not show any drug release at RF OFF. This control study proves that the death of HeLa cell is not associated with any passive leakage or device material, but indeed by wirelessly controlled drug release. Fig. 6(d) and (e) present HeLa cells morphologies at RF turned off (control) and RF turned on for 24 hours incubation periods, respectively. Healthy tumor cells are observed to form a cohesive cluster with well-shaped and smooth surface for RF turned off. The tumor cells, on the other hand, appeared to be rounded and shrunken with some death cells when the RF is turned on. 


\section{Conclusions}

We developed a wirelessly activated and soft drug delivery device. The prototype was designed, fabricated and demonstrated with drug release operation triggered by an external RF magnetic field. The electroactive IPMC cantilever was integrated with a planar LC receiver circuit consisting of a resonator and load loops. The cantilever serves as the actuator to control the opening gap of the release orifice using input power from the transmitter. The wireless coupling effects of the encapsulated LC receiver circuit were examined and analyzed, including resonant frequency study in multiple media and position orientation at the transmitter circuit. The IPMC cantilever deflection and force profile were characterized at different RF voltages and repeated RF ON-OFF cycles. A proof-of-concept release of Cisplatin drug from the fabricated prototype shows successful decrease of cell viability of HeLa cell with RF turned on. The future work will encompass the optimization of the device design and the further miniaturization of the device for lower loss and higher power transfer efficiency, as well as an in-vivo study in animals.

\section{Conflicts of interest}

There are no conflicts to declare.

\section{Acknowledgements}

This work was supported by university grant, UTARRF (IPSR/RMC/UTARRF/2017-C1/C01, Vote: 6200/CC8 and IPSR/RMC/UTARRF/2017-C2/T07).

\section{Notes and references}

1 S. Ward, V. Foroutan, R. Majumdar, O. Mahdavipour, S. A. Hussain and I. Paprotny, IEEE transactions on nanobioscience, 2015, 14, 323-331.

2 S. A. Hussain, S. Ward, O. Mahdavipour, R. Majumdar and I. Paprotny, Next-Generation Robotics II; and Machine intelligence and Bio-inspired Computation: Theory and Applications IX, Int. Society for Optics and Photonics, 2015, 9494.

3 A. Hamie, E. Ghafar-Zadeh and M. Sawan, in IEEE Int. Symp. on Medical Measurements and Applications Proceedings (MeMeA), QC, Canada, 2013, pp. 278-281..

4 P.-Y. Li, J. Shih, R. Lo, S. Saati, R. Agrawal, M. S. Humayun, Y.-C. Tai and E. Meng, Sensors and Actuators A: Physical, 2008, 143, 41-48.

5 R. Lo, K. Kuwahara, P.-Y. Li, R. Agrawal, M. S. Humayun and E. Meng, in IEEE Int. Conf. on Microtechnologies in Medicine and Biology, Okinawa, Japan,2006, pp. 74-77.

6 R. Farra, N. F. Sheppard, L. McCabe, R. M. Neer, J. M. Anderson, J. T. Santini, M. J. Cima and R. Langer, Science translational medicine, 2012, 4, 122ra121-122ra121.

7 N. Elman, H. H. Duc and M. Cima, Biomedical microdevices, 2009, 11, 625-631.

8 H. Gensler, R. Sheybani, P.-Y. Li, R. Lo, S. Zhu, K.-T. Yong, I. Roy, P. N. Prasad, R. Masood and U. K. Sinha, in IEEE 23rd Int. Conf. on Micro Electro Mech. Syst., Hong Kong, China, 2010, pp. 23-26.

9 F. N. Pirmoradi, K. Ou, J. K. Jackson, K. Letchford, J. Cui, K. T. Wolf, F. Gräber, T. Zhao, J. A. Matsubara and H. Burt, M. Chiao, L. lin, In IEEE 26th Int. Conf. on Micro Electro Mech. Syst. (MEMS 2013), Taipei, Taiwan,2013, pp. 1-4.

$10 \mathrm{~J}$. Maloney and J. Santini, in 26th Annual Int. Conf. of the IEEE Engineering in Medicine and Biology Society (IEMBS'04), San Francisco, USA, 2004, pp. 2668-2669.

11 Z. Sbiaa, in28th Annual International Conference of the IEEE Engineering in Medicine and Biology Society (EMBS'06), NY, USA, 2006, pp. 5621-5624.

12 A. T. Evans, S. Chiravuri and Y. B. Gianchandani, in IEEE 22nd Int. Conf. on Micro Electro Mech. Syst., Sorrento, Italy,2009, pp. 252-255.

13 M. Zainal and M. M. Ali, in IEEE EMBS Conf. on Biomedical Engineering and Sciences (IECBES), Kuala Lumpur, Malaysia,2016, pp. 76-79.

14 S. Rahimi and K. Takahata, in IEEE 24th Int. Conf. on Micro Electro Mech. Syst. (MEMS), Cancun, Maxico, 2011, pp. 1019-1022.

15 P. S. Chee, M. N. Minjal, P. L. Leow and M. S. M. Ali, Sensors and Actuators A: Physical, 2015, 233, 1-8.

16 B.-K. Fang, M.-S. Ju and C.-C. K. Lin, Sensors and Actuators A: Physical, 2007, 137, 321-329.

17 J. S. Lee, S. Gutta, W. Yim and K. J. Kim, in/EEE/ASME Int. Conf. on Advanced Intelligent Mechatronics (AIM), Montreal, Canada, 2010, pp- 157-162.

18 E. Y. Chow, M. M. Morris and P. P. Irazoqui, IEEE Microwave Magazine, 2013, 14, 64-73.

19 K. Abdelnour, A. Stinchcombe, M. Porfiri, J. Zhang and S. Childress, IEEE/ASME Transactions on Mechatronics, 2012, 17, 924-935.

20 L. Zhang, Y. Yang and C. Soh, in Smart Materials in Structural Health Monitoring, Control and Biomechanics, Springer, 2012, pp. 533-567.

21 S. Lee and K. J. Kim, Smart materials and structures, 2006, 15, 1103.

22 T. T. Nguyen, N. S. Goo, V. K. Nguyen, Y. Yoo and S. Park, Sensors and Actuators A: Physical, 2008, 141, 640-648. 
23 M. S. S. Mousavi, A. H. Karami, M. Kolahdouz, F. Manteghi and F. Ataei, Journal of the mechanical behavior of biomedical materials, 2018.

24 H. R. Cheong, C. Y. Teo, P. L. Leow, K. C. Lai and P. S. Chee, Smart Materials and Structures, 2018, 27, 055014.

25 F. N. Pirmoradi, J. K. Jackson, H. M. Burt and M. Chiao, Lab on a Chip, 2011, 11, 2744-2752.

26 H. Lei, W. Li and X. Tan, Sensors and Actuators A: Physical, 2014, 217, 1-12.

27 M. Shahinpoor and K. J. Kim, Smart materials and structures, 2001, 10, 819.

28 Y. Zhang, C. Ma and L. Dai, in 2nd IEEE Int. Conf. on Nano/Micro Engineered and Molecular Systems (NEMS'07), Bangkok, Thailand,2007, pp. 68-71.

29 S. Ford, G. Macias and R. Lumia, Smart Materials and Structures, 2014, 24, 025015.

30 A. Abid, J. M. O’Brien, T. Bensel, C. Cleveland, L. Booth, B. R. Smith, R. Langer and G. Traverso, Scientific reports, $2017,7,46745$.

31 M. Zainal, A. Ahmad and M. M. Ali, Biomedical microdevices, 2017, 19, 8

32 J. Geist, J. J. Shah, M. V. Rao and M. Gaitan, Journal of research of the National Institute of Standards and Technology, 2007, 112, 177.

33 R. Majumdar, V. Foroutan and I. Paprotny, in IEEE 28th Int. Conf. on Micro Electro Mech. Syst., Estoril, Portugal, 2015.

34 R. Majumdar and I. Paprotny, Journal of Microelectromechanical Systems, 2017, 26, 671-678.

35 J. Fong, Z. Xiao and K. Takahata, Lab on a Chip, 2015, 15, 1050-1058.

36 Y. Yi, U. Buttner and I. G. Foulds, Lab on a Chip, 2015, 15, 3540-3548.

37 H.-P. Hohn, U. Steih and H.-W. Denker, In Vitro Cellular \& Developmental Biology-Animal, 1995, 31, 37-44.

38 E. Varoni, M. Tschon, B. Palazzo, P. Nitti, L. Martini and L. Rimondini, Connective tissue research, 2012, 53, 548-554.

39 T. Karacolak, A. Z. Hood and E. Topsakal, IEEE Transactions on Microwave Theory and Techniques, 2008, 56, 10011008.

40 P. Soontornpipit, C. M. Furse and Y. C. Chung, IEEE Transactions on Microwave theory and techniques, 2004, 52, 1944-1951.

41 T. Karaçolak, Mississippi State University, 2009.

42 M. Horky, G. Wurzer, V. Kotala, M. Anton, B. Vojtesek, J. Vácha and J. Wesierska-Gadek, Journal of Cell Science, $2001,114,663-670$.

43 C. H. Lee, H. Kim, D. V. Harburg, G. Park, Y. Ma, T. Pan, J. S. Kim, N. Y. Lee, B. H. Kim and K.-I. Jang, NPG Asia materials, 2015,7, e227.

44 F. Ordikhani, M. Erdem Arslan, R. Marcelo, I. Sahin, P. Grigsby, J. K. Schwarz and A. K. Azab, Pharmaceutics, 2016, 8, 23. 